\title{
Intelligent Cell Phone Detector System at 4g Bands Of Frequencies
}

\author{
Himanshu Verma ${ }^{1}$, Rudra Dev Tiwari ${ }^{2}$, Sarvesh Mishra ${ }^{3}$, Shubham Srivastava ${ }^{4}$, \\ Amit Singh $^{5}$, Harikesh Tripathi ${ }^{6}$ \\ 1,2,3,4,5,6 Department of ECE/United College of Engineering \& Management/Dr.A.P.J. Abdul Kalam Technical \\ University, Allahabad, India
}

\begin{abstract}
This handy, pocket-size mobile transmission detector or sniffer can sense the presence of an activated mobile cell phone from a distance of one and-a-half meters. So it can be used to prevent use of mobile phones in examination halls, confidential rooms, etc. It is also useful for detecting the use of mobile phone for Spying and unauthorized video transmission. The circuit can detect the incoming and outgoing calls, SMS and video transmission even if the mobile phone is kept in the silent mode. The moment the Bug detects RF transmission signal from an activated mobile phone, it starts sounding a beep alarm and the LED blinks. The alarm continues until the signal transmission ceases. Assemble the circuit on a general purpose PCB as compact as possible and enclose in a small box like junk mobile case. Carefully solder the capacitor in standing position with equal spacing of the leads. The response can be optimized by trimming the lead length of $C 3$ for the desired frequency. You may use a short telescopic type antenna. The use of the cell phone has made communication easier and faster, integrating the world into a global village as people who are in different geographic location are connected in seconds, it's great to be able to call anyone at any time. There is great need to limit the use of cell phones at particular places and at particular time. Hence, the use of intelligent mobile phone detector is needed. This work concentrates in designing a system that will dictate the presence of GSM signals from an unauthorized user in restricted areas.
\end{abstract}

Keywords: Cell Phone Detector, GSM signal, Communication restricted area, IC CA3130, 555 Timer IC, Buzzer, LED.

\section{Introduction}

Cell phone technology is one that is ubiquitous and is progressing by every passing day. The rapid use of cell phone at the beginning of the 21 st century eventually raised problem such as their potential use to invade privacy of contributes to increase cheating. In addition public backlash was growing against the intrusive disruption cell phone. The older analog cell phones often effected from poor reception and could even be failed by common interfacing condition such as high frequency noise. Increasing digital phones have led to more elaborated counters. Intelligent cell phone devices are option to more expensive measures against denial of service providers. This system will indicate the presence of GSM signals from an unauthorized person in prohibited areas. The cell phone detector is an advanced device which can be used in the fields of communication, surveillances etc. [1]

A very common scenario is one of the students operating cell phone in examination halls where its use is obviously restricted. The examiners have to personally go to the student and check his pocket or desks for any cell phone. With the help of cell phone detector the examiner will know if a cell phone is active and can take action immediately.[2]The intelligent mobile phone detection project is an advanced device which finds various applications in the modern fields of communication and surveillances. This work is very useful for the private meetings, examination halls, defence establishments etc ., where the use of an active mobile communication (GSM) device are prohibited.[2]

The first signal detection technique, an existing design utilizing discrete component is difficult to implement. They are very affordable to construct, but require precision tuning. This design is analyzed and found to be inaccurate.[4]The second signal detection technique, a design using a down converter, voltage controlled oscillator (VCO), and a bandpass filter was investigated for cellular phone detection. The performance of this technique through hardware and computer modeling is discussed and the results are presented. The new system is accurate and a practical solution for detecting cellular phone in a secure facility.[4] The detector can detect both incoming and outgoing calls, SMS, video transmission in silent mode also. The moment when bug detect RF signals from a cell phone, it starts sounding beep alarm by buzzer and LED starts blinking. A simple RF detector using tunned LC circuit is not correct for detecting signals in the cell phone's frequency band.[3] 


\section{Frequency Range}

The transmission frequency of mobile phones ranges from $900 \mathrm{MHZ}$ to $3000 \mathrm{MHZ}$ with a wavelength of 3.3 to $10 \mathrm{CM}$. So a circuit detecting gigahertz signals is required for a mobile bug [2]. The lead length of the capacitor is fixed as $18 \mathrm{~mm}$ with a spacing of $8 \mathrm{~mm}$ between the lead to get the desired frequency. The disk capacitor along with the leads acts as a small gigahertz loop antenna to collect the RF signal from the mobile phone [7]. The different frequency bands are given below in table 1.

\section{Design Objective And Component}

The objective includes building mobile detector to locate a phone within 1 to 1.5 meters.

Principle used components in the circuits are given below:

\section{1: IC CA3130:}

Op-amp IC CA3130 (IC1) is used in the circuit as a current-to-voltage converter with capacitor C3 connected between its inverting and non-inverting inputs [10]. It is a CMOS version using gate-protected pchannel MOSFET transistors in the input to provide very high input impedance, very low input current and very high speed of performance.[1]

\section{2: 555 Timer IC:}

IC 555 is a highly stable controller capable to producing accurate timing pulses. With the monostable operation the time delay is controlled by one external resistor and one capacitor. With an astable operation the frequency and duty cycle are accurately controlled by two external resistors and one capacitor [8]. These are used in applications like pulse generation, time delay generation etc. CA3130A and CA3130 are op amps that combine the advantage of both CMOS and bipolar transistors. Gate protected P-Channel MOSFET (PMOS) transistors are used in the input circuit to gain high-input impedance, very-low-input current, and exceptional performance [11]. The use of PMOS transistors in the input stage result gives common-mode input-voltage capability down to $0.5 \mathrm{~V}$ below the negative-supply terminal, an important concept in single supply applications.[3]

\section{3: Buzzer:}

A buzzer or beeper is a signaling device, usually electronic, typically used in automobiles, household appliances such as microwave ovens, or game shows. It mostly consists of a number of switches or sensors connected to a control unit that determines if and which button was pushed or a preset time has lapsed, and usually illuminates a light on the appropriate button or control panel, and sounds a warning in the form of a continuous or intermittent buzzing or beeping sound.[5]

\section{4: LED:}

A light-emitting diode (LED) is an electronic light source. LEDs are used as indicator lamps in many kinds of electronics and increasingly for lighting. LEDs work by the effect of electroluminescence,. The LED was introduced as a practical electronic component in 1962. All early devices emitted low-intensity red light, but modern LEDs are available across the visible, ultraviolet and infra red wavelengths, with very high brightness. [7] LEDs are based on the semiconductor diode. When the diode is forward biased (switched on), electrons are able to recombine with holes and energy is released in the form of light. This effect is called electroluminescence and the color of the light is determined by the energy gap of the semiconductor. The LED is usually small in area (less than $1 \mathrm{~mm}^{2}$ ) with integrated optical components to shape its radiation pattern and assist in reflection. LEDs present many advantages over traditional light sources including lower energy consumption, longer lifetime, improved robustness, smaller size and faster switching. However, they are relatively expensive and require more precise current and heat management than traditional light sources [10]. Different colour of LEDs are given below in figure 1.

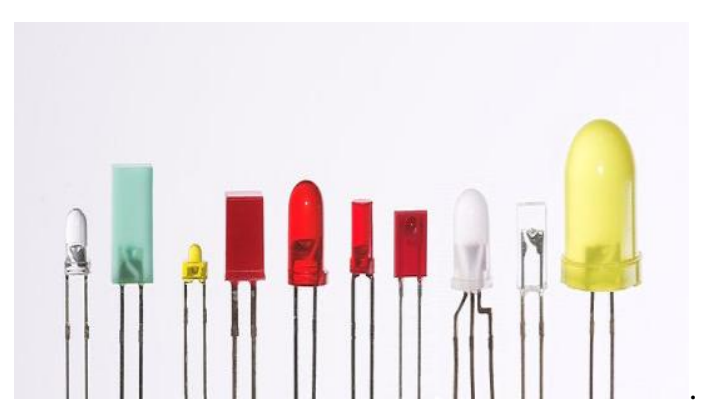

Fig.1 Various types of LEDs 


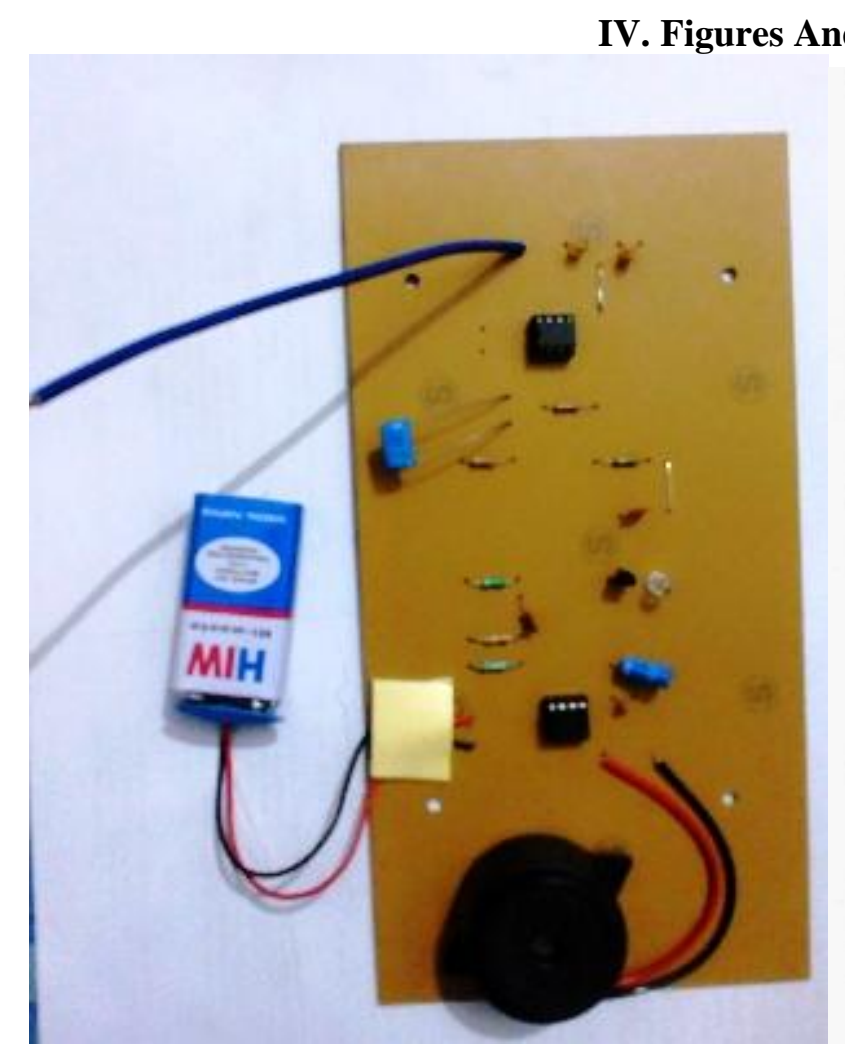

Fig 2: Final circuit diagram,

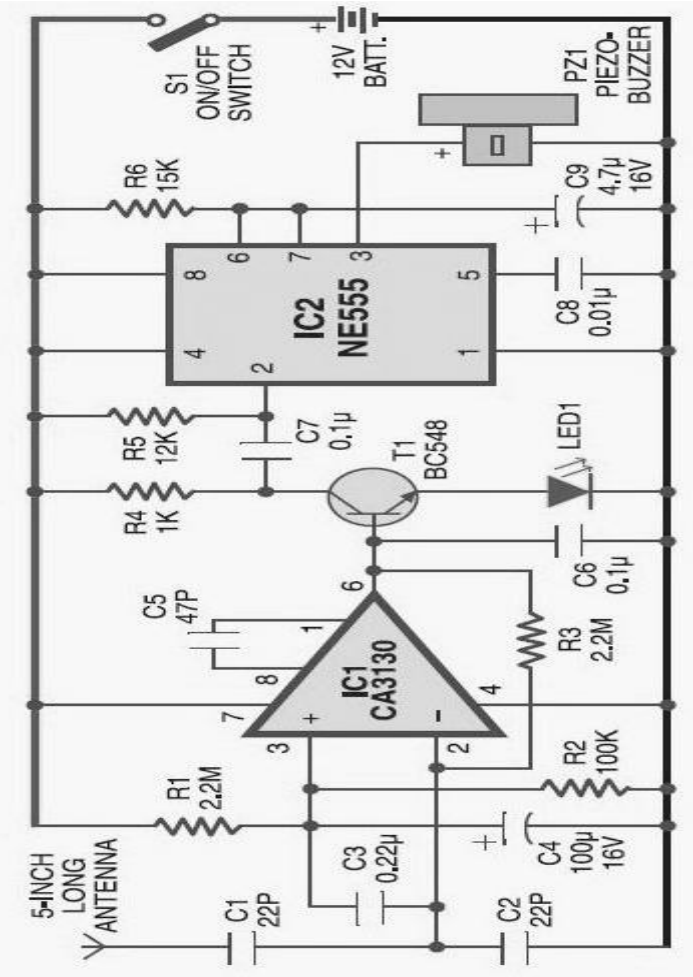

Fig 3: Systematic circuit diagram

\begin{tabular}{|l|c|l|}
\hline S.NO. & NETWORK & BAND \\
\hline 1 & $2 G$ & $\begin{array}{l}\text { GSM 900 } \\
\text { GSM1800 }\end{array}$ \\
\hline 2 & $3 G$ & $\begin{array}{l}\text { UMTS 900 } \\
\text { UMTS 2100 }\end{array}$ \\
\hline 3 & $4 G$ & $\begin{array}{l}\text { LTE } 850 \\
\text { LTE 1800 } \\
\text { LTE 2100 } \\
\text { LTE 2600 }\end{array}$ \\
\hline
\end{tabular}

Table 1: Network and their Frequency band

\section{Working Principle}

When the mobile phone signal is detected by $\mathrm{C} 3$, the output of IC1 becomes high and low alternately according to the frequency of the signal as indicated by LED1. This triggers mono stable timer IC2 through capacitor C7. Capacitor C6 maintains the base bias of transistor T1 for fast switching action. The low-value timing components R6 and C9 produce very short time delay to avoid audio nuisance. When the power is switched on, as stated above, IC1 will give a high output and T1 conducts to trigger LED and Buzzer .This can be a good indication for the working of the circuit. The circuit can detect both the incoming and outgoing calls, SMS and video transmission even if the mobile phone is kept in the silent mode [4]. The moment the bug detects RF transmission signal from an activated mobile phone, it starts sounding a beep alarm and the LED blinks. The alarm continues until the signal transmission ceases. An ordinary RF detector using tuned LC circuits is not suitable for detecting signals in the $\mathrm{GHz}$ frequency band used in mobile phones. The transmission frequency of mobile phones ranges from 0.9 to $3 \mathrm{GHz}$ with a wavelength of 3.3 to $10 \mathrm{~cm}$. So a circuit detecting gigahertz signals required for a mobile bug [9]. Circuit diagram is given above in figure 2 . 
STEP 1- Start

\section{Algorithm}

STEP 2- Check the circuit and the component properly

STEP 3- Make a call by cell phone

STEP 4- RF signals captured by capacitor C3

STEP 5- The RF signals passes through op amp IC CA 3130 and 555 Timer IC

STEP 6- When signal is detected then LED starts blinking and Buzzer starts alarming sound

STEP 7- If signal is not detected then there will be no indication in any form

STEP 8- End

\section{Flow Chart}

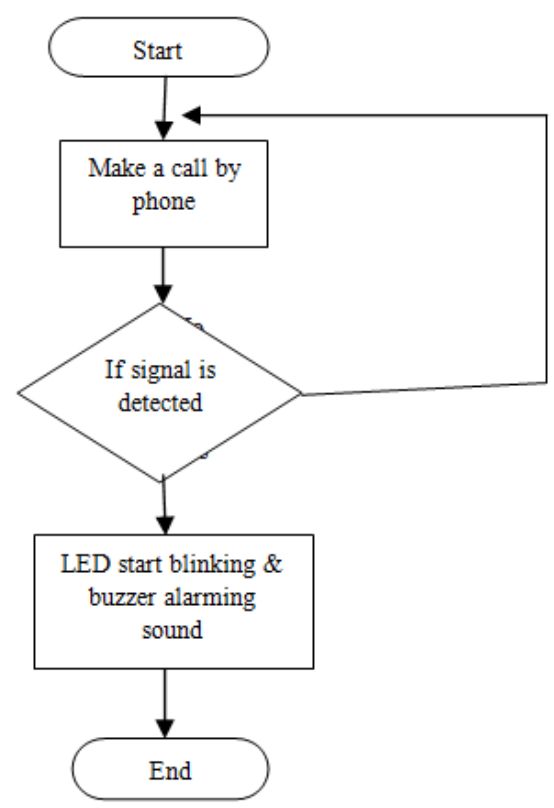

\section{Future Scope}

Trying to increase the detecting range of mobile bug to few more meters for observing wide ranges of area. In the future time this detector will be improved in all ways. The prototype version has only limited range of 2 meters. But if a preamplifier stage using JFET or MOSFET transistor is used as an interface between the capacitor and IC, range can be increased.

\section{Conclusion}

This pocket-size mobile transmission detector or sniffer can sense the presence of an activated mobile cellphone from a distance of one and-a-half meters [8]. So it can be used to prevent use of mobile phones in examination halls, confidential rooms, etc. It is also useful for detecting the use of mobile phone for spying and unauthorized video transmission. [10]

In this paper we made an attempt to design a mobile detector which can detect both the incoming and outgoing calls as well as video transmission even if the mobile is kept at the silent mode [6]. Our circuit has detected the presence of an active mobile phone even at a distance of about one and half a meter. It gave the indication of an active mobile phone by glowing The LED, according to the receiving frequency and by buzzing the sound of the buzzer. The alarm continues until the signal is ceases. [9]

\section{Acknowledgements}

We are highly obliged for the indispensable efforts made by our Head of the Department Prof.(Dr.) Nandita Pradhan. We are also thankful to the faculty member and lab instructors specially Mr. Rohit Shrivastava of ECE, UCEM for constant constructive criticism and valuable suggestions, which benefited us a lot while developing the project on " Mobile Phone Detector". 


\section{References}

[1]. Christian C. Mbaocha: Design and Implementation of Intelligent Mobile Phone Detector; Acadmic Research International, ISSN:2223- 9944 Vol 3, No 1, July 2012

[2]. Tanvi Deshpande \& Nakul Jadhav: Active cell phone detection and display using Atmega-88 Microcontroller, International Journal of Reaserch in Engineering \& Technology; ISSN(E):2321-8843; ISNN(P):2347-4599 Vol. 3, Issue 8, Aug 2015

[3]. Novel mobile detector sensing alarming and reporting system K. Mohan Dece, ARPN Journal of science and technology SRM University VOL.2,NO.1, Jaunary 2012

[4]. Nicholas W. Scott : Study of Cellular phone detection techniques ; University of Nebraska; April 2011

[5]. Mohan Kumar, D. 2008. "Mobile Bug". Electronics for you magazine. www.espow.com/jammers/security- surveillance

[6]. Abdul K.A, Asa ${ }^{e} d$ Nalm, Ahmed Hassan and Ayman Samier, 2008. Mobile phone intelligent jamming system. Jordan

[7]. Christian C.M. 2012. "Design and Implementation of Intelligent Mobile Phone Detector". Academic Research International. Vol. 3, No. 1

[8]. Ahmed, S.A., Ahmed N.R.M. 2006. Dual band mobile jammer for GSM900 and BSM1800

[9]. B. Kainka "Mobile Phone Sniffer track down mobile phones using this handy directional finder"

[10]. Cell Phone Detection Techniques, U.S. Department of Energy. October 2007. Accessed January, 2010

[11]. Cell Phone Detector, Circuit-Projects.com Quality Circuits Archive 